\title{
Search for ultra-long gravitational waves in pulsars' rotational parameters
}

\author{
Maxim S. Pshirkov \\ Pushchino Radio Astronomy Observatory, ASC LPI, Pushchino, 142290, Russia \\ email: pshirkov@prao.ru
}

\begin{abstract}
A method is suggested to explore the gravitational wave background (GWB) in the frequency range from $10^{-12}$ to $10^{-8} \mathrm{~Hz}$. That method is based on the precise measurements of pulsars' rotational parameters: the influence of the gravitational waves $(\mathrm{GW})$ in the range will affect them and therefore some conclusions about energy density of the GWB can be made using analysis of the derivatives of pulsars' rotational frequency. The calculated values of the second derivative from a number of pulsars limit the density of GWB $\Omega_{g w}$ as follows: $\Omega_{g w} h^{2}<10^{-6}$. Also, the time series of the frequency $\nu$ of different pulsars in pulsar array can be cross-correlated pairwise in the same manner as in anomalous residuals analysis thus providing the possibility of GWB detection in ultra-low frequency range.
\end{abstract}

Keywords. gravitational waves, (stars:) pulsars: general, methods: data analysis

Propagation of pulsar signal in space-time perturbed by a stochastic gravitational wave field results in apparent deviations of pulsar rotational frequency; also gravitational waves affect the derivatives of pulsars rotational frequency - these values absorb the effect caused by gravitational waves with ultra long wavelengths $\left(\sim(c D)^{-1}<f_{g w}<T_{\text {obs }}^{-1}\right)$.

Precise pulsar timing allows us to measure second derivative of rotational frequency for a number of pulsars. It is plausible to regard the calculated value of $\ddot{\nu}$ as caused by unknown factors that are intrinsic or extrinsic to pulsar. For detailed calculations see Pshirkov (2009). We can write down the final result:

$$
\Omega_{g w}<\frac{T_{\mathrm{obs}}^{2}}{2 \pi^{2} H_{0}^{2}}\left(\frac{\ddot{\nu}_{\mathrm{obs}}}{\nu_{0}}\right)^{2}
$$

It is instructive to make some numerical estimates using the data for PSR B1937+21:

$$
\Omega_{g w} h^{2}<10^{-6}
$$

Also, the time series of the frequency $\nu$ from different pulsars in pulsar array can be cross-correlated pairwise. Comparison of angular dependence of obtained functions with known sample of GWB induced correlation similarly to usual pulsar timing (see Jenet et al. (2005)) residual analysis makes possible to detect GWB in the frequency range of our interest. That method can probe GWB down to energy density $\Omega_{g w} h^{2}<10^{-7}$.

\section{Acknowledgements}

I would like to thank the support by RFBR grants no. 09-02-00922 and 07-02-01034.

\section{References}

Jenet, F. A., Hobbs, G. B., Lee K. J., \& Manchester R. N. 2005, ApJ, 625, L123

Pshirkov, M. S., MNRAS, DOI: 10.1111/j.1365-2966.2009.15221.x 